\title{
Development of a Neuro-Fuzzy Diagnostic System Mathematical Model for Internal Combustion Engines
}

\author{
Lenar A. Galiullin*1, Rustam A. Valiev ${ }^{2}$, Lejsan B. Mingaleeva ${ }^{3}$ \\ ${ }^{1,2,3}$ Kazan Federal University, Naberezhnye Chelny Institute \\ *Email: galilenar@yandex.rutel.
}

\section{Received: 21st October 2017 Accepted: 16th November 2017, Published: 31st December 2017}

\begin{abstract}
The methods are given for an adaptive engine control system creation in this paper for tests and diagnostics based on a neuro-fuzzy system, which made it possible to simplify the elements of an automated system structure for the testing and the diagnosing of internal combustion engines. The system created provides a more accurate method of controlling certain parameters of the engine, which improved the quality of control when it was diagnosed and allowed to reduce the volume of the knowledge base.
\end{abstract}

In order to control the engine during its testing and diagnostics it is proposed to use the methods of neurofuzzy inference, widely applied in the development of intelligent systems. In order to describe an engine management, expert knowledge is used instead of mathematical models.

The control of internal combustion engines (ICE) in ASID based on a neural-fuzzy system is founded on a knowledge base containing a number of fuzzy rules automatically generated by a neural network. The filling in of this knowledge base is also possible by direct measurement method, but it is more appropriate to fill in the knowledge base with a neural network.

The use of a neural-fuzzy system in ASID allowed the introduction of the initial information - the knowledge base of fuzzy control rules into the control system. The proximity of the form of control rule forms to natural language makes it possible to work with the knowledge base for test technologists.

Keywords: Mathematical Model, Internal Combustion Engine, Neuro-Fuzzy System, Automation.

\section{Introduction}

Fuzzy logic systems and neural networks are the most important application of intelligent systems. The functioning of such systems differs from the functioning of classical systems [1].

The main goal of the neuro-fuzzy system is to control the external impact, in which an object is controlled by fuzzy rules [2].

The control system of an internal combustion engine based on the neuro-fuzzy system can be represented as a set of modules (Fig. 1).

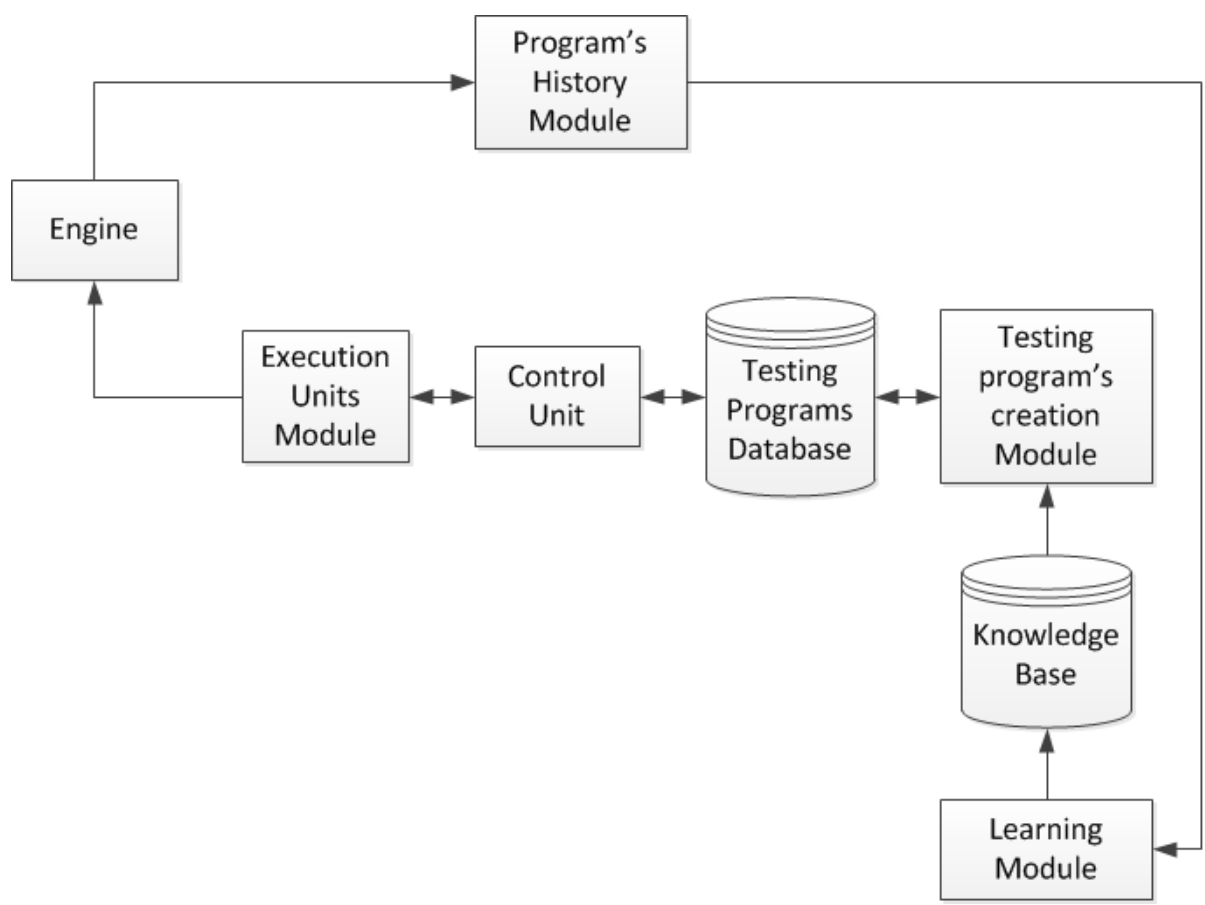

Fig. 1. The Structure of the Neural-Fuzzy Engine Diagnostics System

The ASID of internal combustion engines includes the following modules: "Program's History Module",
"Learning Module", "Knowledge Base", "Testing program's creation module", "Control Unit", 
"Execution Units Module", "Testing Programs Database", "Engine".

A technologist creates a knowledge base for fuzzy control rules, on the basis of which a control program is created using ASID ICE [3].

A user creates the sequences to change the parameters of interest in the "Testing program's creation Module" that are recorded from time to time in the "Testing Programs Database" unit. In this multi-level database the input images are transformed sequentially into control actions through a fuzzy output system, which is located in the "Testing program's creation Module". In order to obtain control values, the fuzzy system applies fuzzy control rules and linguistic variables that are in the "Knowledge Base" unit. Eventually, the sequence of control actions is created, which is stored in the Testing Programs Database. Based on the received data, the "Control Unit" controls the ACID ICE directly, for example, changes the amount of fuel supplied to an engine or external load. During the tests with the "Execution Units Module" information is generated about the current status of a control object, for example, about the crankshaft speed, torque, fuel

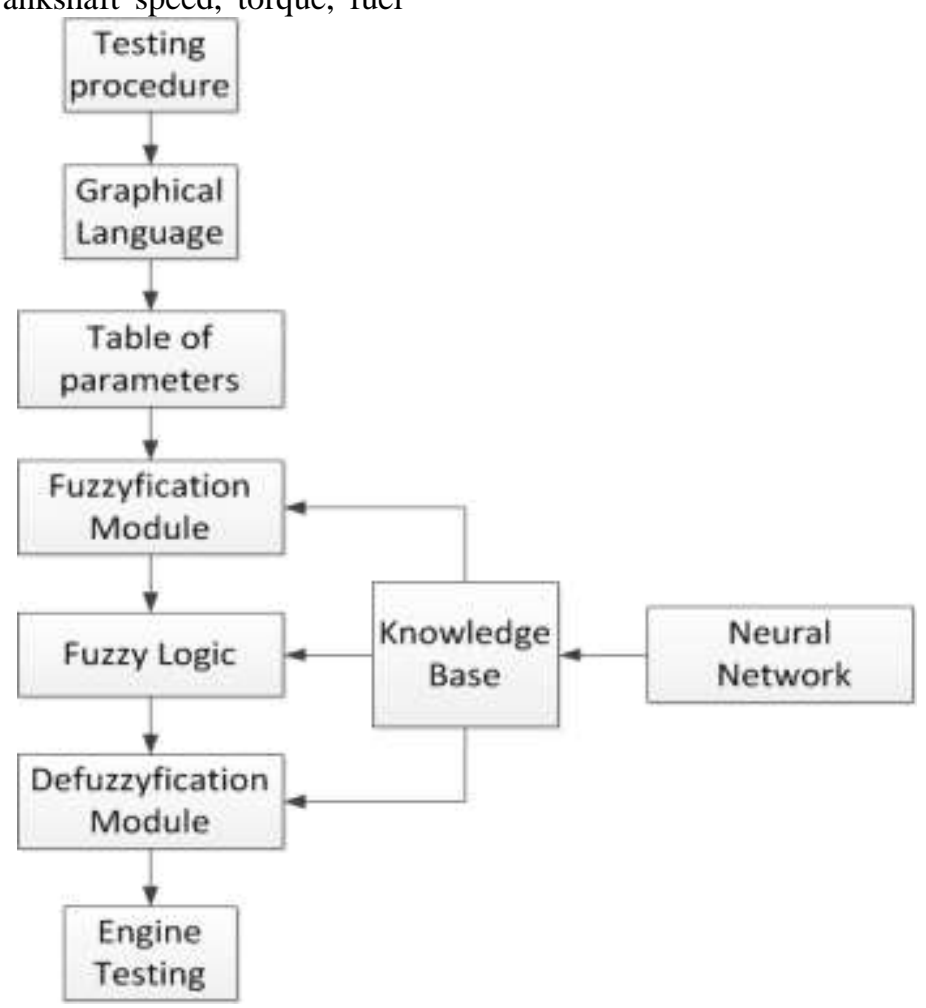

Fig. 2. Structure of the Engine Test Process consumption, etc. The listed data is stored in the "Program's History Module". The incoming data are also directly transferred to the "Control Unit" from the sensors, which is necessary to prevent emergencies. The ASDIC ICE stops its operation in the event of an engine speed sudden increase, oil temperature increase or in other cases.

In "Learning Module" feedback is received from the "Program's History Module". This feedback is necessary to implement the principle of adaptive management, as well as to fill in the knowledge base in which the management rules are located. These rules can also be created or adjusted on the basis of direct measurement method by the analysis of the information coming from the "Program's History Module" in the "Learning Module".

\section{Methods}

The process of engine test procedure development based on the developed structure using a fuzzy system is represented by the following sequence of steps shown on Fig. 2.
The upper level is the test procedure, namely the document regulating the conduct of any type of engine testing.

It reflects the main points of tests - a purpose and a designation, the volume, the state of a test object choice, the test equipment, the test conditions and methodology, the methods of result evaluation, the requirements for technical characteristics and fire safety. The test procedure is developed by a technologist taking into account the engine parameters of interest. When it is used, a technologist must specify the parameters of the engine that would be desirable to obtain at the output, and ASID will select the optimal input control actions for the implementation of these modes.

At another stage this testing procedure is presented in the form of images - graphic images. An image is a sequence of varying modes, each of which can be represented as a segment. The time of an engine operating mode is equal to the projection on the abscissa axis. The change of the controlled parameter during this time interval corresponds to the projection 
on the ordinate axis.

An example of image changes in the engine operation by the engine speed is shown on Fig. 3. When an image is created, interactive and cognitive graphics are used, which allows a technologist to adjust the engine operating modes independently, without ASID programming. One of the advantages of cognitive graphics is its simplicity, so a technologist imagines the tests being conducted better and sets the control actions graphically at the design stage to exclude emergency modes.

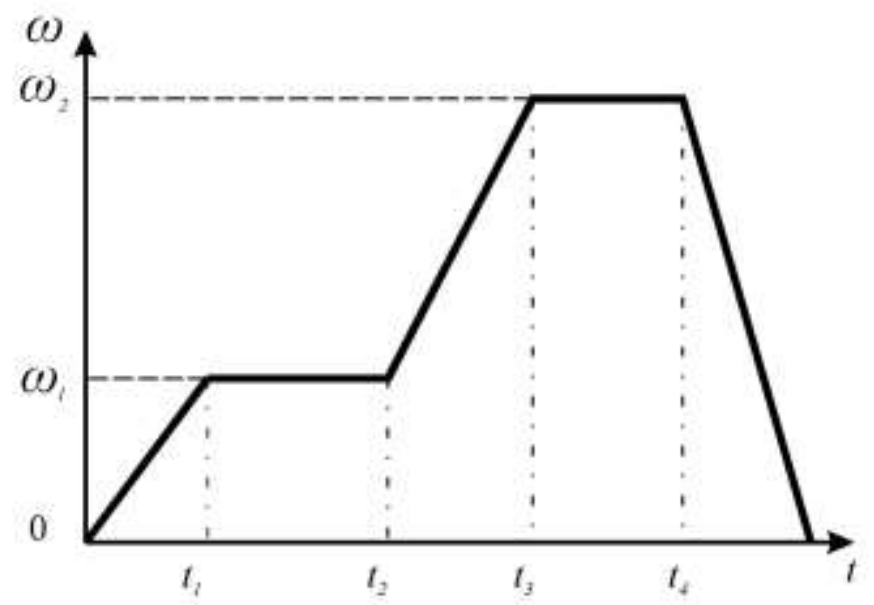

Fig. 3. The Graph of the Engine Operating Conditions According to the Crankshaft Speed

\section{Results and Discussion}

One of the main components of the intelligent ASID ICE system is the knowledge base. The knowledge base ASID ICE is represented by a set of facts and output rules, allowing building a logical conclusion and conducting the processing of incoming information [4].

The ASID knowledge base consists of three levels (Fig. 4).

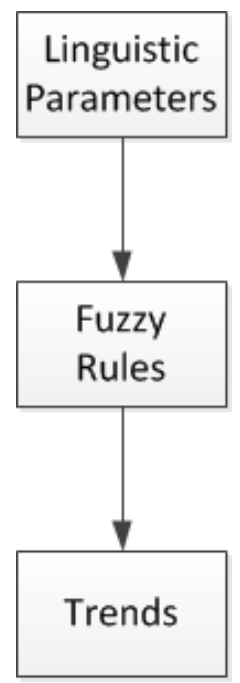

Fig. 4. Knowledge Base

Linguistic variables are located at the first level. These variables are set by a technologist at the testing stage for a particular engine model. They should include the parameters by which you can manage and install tests. Linguistic marks are formed by test technologists or by direct measurements. This level defines the basic range and the number of linguistic variables on it, as well as the type of belonging functions. The number of linguistic variables determines the accuracy of the received control action. The improvement of control depends on the number of markings increase, but at the same time the time of filling in the knowledge base increases. The syntactic and semantic rules of linguistic variables are obtained at this level [5].

The second level of the knowledge base consists of fuzzy control rules used to convert the specified parameters into a control action. These rules are made up of linguistic marks, defined at the previous level. Fuzzy rules can be formed by test technologists, by direct measurement and by a neural network use with self-learning. During the use of a neural network with 
self-learning, an automatic process of fuzzy rules knowledge base development occurs. This level also contains semantic rules that set the real engine test modes. For example, it seems impossible to generate maximum power and a torque at very low engine speed. The specified fuzzy control rules also allow the description of emergency modes.

The third level includes the priority vectors set for characteristic rating. The vectors of priorities consist of the parameters that must be set by [6]. On their basis, you can establish a certain direction of testing. For example, the tests for environmental friendliness, power or an engine operation economy. This level is set by the method of expert assessments.

During the diagnostics of engine faults with ASID the feedback is used [7]. Feedback is used both to correct the results and develop a control vector, and for a knowledge base development.

When the knowledge base is filled in with dynamic engine test modes, the control rule will have the following form:

\section{IF $\omega_{i}$ AND $R_{j}$ THEN $h_{i}$;}

where $\omega_{i}$ - engine speed $\left(\omega_{1}, \omega_{2}, \omega_{3}, \ldots, \omega_{n}\right)$; $R_{1} \ldots ; R_{r}$ $R_{j}$ - engine operation mode $R_{-r} ; \ldots R_{-1} ; R_{0}$;

$h_{i}-$ the position of HPFP rail (for a diesel engine) $h_{1}, h_{2}, \ldots h_{n}$.

Based on such fuzzy control rules, it is possible to form a knowledge base for an engine operation in stationary modes [8]. In this case, the $R_{j}$ mode will have a constant value of $R_{0}$, i.e. an engine will be operated in the mode in which neither speed reduction or increase occurs. The basis of stationary modes will be the following one:

\section{IF $\omega_{i}$ AND $R_{0}$ THEN $h_{k}^{x}$,}

where $\omega_{i}$ - engine speed $\left(\omega_{1}, \omega_{2}, \omega_{3}, \ldots, \omega_{n}\right)$, $R_{0}$ - stationary mode of an engine operation,

$h_{k}$ - HPFP regulator position $\left(h_{l}, h_{2}, \ldots h_{l}\right)$.

The operating mode $\mathrm{R}_{\mathrm{j}}$ arises during the use of direct measurement method. Let's suppose that the control rule is given:

$$
\text { IF } \omega_{k} \text { AND } R_{l} \text { THEN } h_{x}{ }^{x},
$$

where $\omega_{k}, R_{l}$ are fixed fuzzy labels of the levels $\omega_{i}$ and
$R_{j}$

$h_{x}$ the unknown value of $h_{k}$ level.

In order to fill this rule, the following nearest rule is sought:

$$
\text { IF } \omega_{k} \text { AND } R_{0} \text { THEN } h_{s},
$$

where $\omega_{k}, R_{l}, h_{s}$ are fixed fuzzy marks for the levels $\omega_{i}$, $R_{j}$ and $h_{k}$.

Based on the method of direct measurements, two new control rules are created:

(1) IF $\omega_{k}$ AND $R_{l}$ THEN $h_{s}$;

(2) IF $\omega_{k}$ AND $R_{l}$ THEN $h_{s+l}$.

The control value of the rule (1) is the same as the closest precedent, and for rule (2) the nearest fuzzy mark is placed in the direction of $R_{j}$ mode change (if $R_{j}$ mode of the new rule is better than the previous one, then the reference value is shifted towards the nearest fuzzy mark value, otherwise it decreases) [9].

The result of the first rule work is the values that completely coincide with the precedent. Next, the result of the second rule work is checked in the selfstudy mode. If the first rule turns out to be more accurate, it is left in the knowledge base and the development of the knowledge base is completed. Then rule (2) is replaced by rule (1). Rule (2) is created again based on the basis of the following linguistic variable:
$R_{l}$ THEN $h_{s+l}$;
$R_{l}$ THEN $h_{s+2}$.
(2) IF $\omega_{k}$ AND

(1) IF $\omega_{k}$ AND

These two rules are checked for validity. If rule (1) is more accurate, then it is left in the knowledge base, otherwise the process similar to the previous one, takes place [10].

If after the selection, the most optimal rule does not satisfy the required accuracy, it is necessary to enter the mark of the intermediate fuzzy control parameter based on the intersection operation. If the base value can be associated with an equal degree of probability by two adjacent marks $a_{i}$ and $a_{i+1}$, then a new linguistic label $a_{i, i+l}$ is created at that point.

Graphically, the intersection of linguistic variables is presented on Fig. 5. 


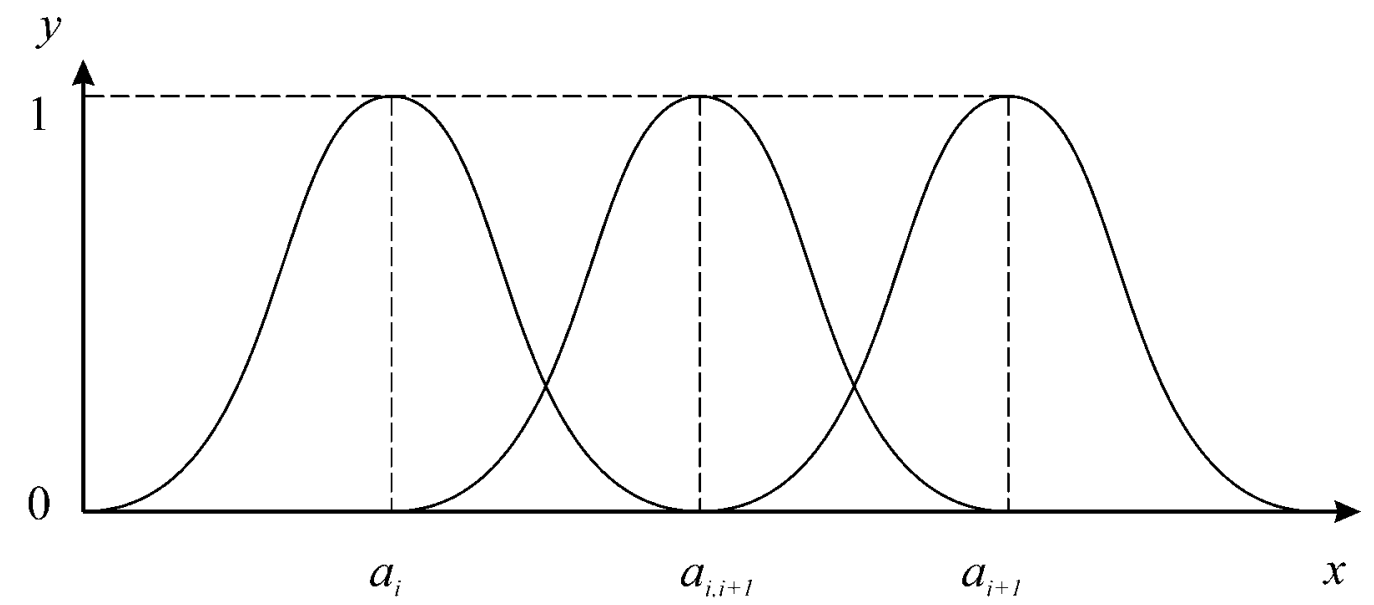

Fig. 5. Intersection of Linguistic Parameters $a_{i}, a_{i+1}$

The introduction of a new linguistic variable will create a new rule of management:

\section{IF $\omega_{k}$ AND $R_{l}$ THEN $h_{s+1, s+2}$.}

If this rule satisfies the required accuracy, then it is stored in the knowledge base, otherwise another fuzzy mark is formed in the range between fuzzy marks with the smallest error.

The number of fuzzy marks is determined by the capabilities of the neural-fuzzy system, since the increase of marks can affect the calculations and the engine control in real time.

\section{Conclusions}

The application of the graphical representation of control actions is convenient for a technologist, which is better than the setting of control actions in the form of numerical data. The next step is the conversion of images into a summary table of parameters. To do this, the entire testing time is divided into time intervals $t_{\text {min }}$, on which an engine will operate. The choice of this value should be as small as possible, as this leads to improved control accuracy and the elimination of emergencies, but as the intervals decrease, computational resources are required. Based on these values, the number of points is set in which control will be conducted during testing:

$S=t_{i s} / t_{\text {min }}$

where $S$ - the number of operation key points;

$t_{i s}$ - total operating time in a given mode;

$t_{\text {min }}$ - the time between two modes of operation.

During the next step, the values of the measured parameters $\left(P_{1 i}, P_{2 i}, P_{3 i}, \ldots, P_{m i}\right)$ are determined for each time interval, where $P_{i}$ are parameter values at a certain time, which are obtained from the corresponding $\mathrm{m}$ graphic images. This data is stored in the parameter table (Table 1).

Table 1 Graphical Parameters of Engine

\begin{tabular}{|c|c|c|c|c|c|c|}
\hline № & Running Time & Graph 1 & Graph 2 & Graph 3 & $\ldots$ & Graph $k$ \\
\hline 1. & $t=0$ & $P_{11}$ & $P_{21}$ & $P_{31}$ & $\ldots$ & $P_{k 1}$ \\
\hline 2. & $t=t_{\min }$ & $P_{12}$ & $P_{22}$ & $P_{32}$ & $\ldots$ & $P_{k 2}$ \\
\hline 3. & $t=t+t_{\min }$ & $P_{13}$ & $P_{23}$ & $P_{33}$ & $\ldots$ & $P_{k 3}$ \\
\hline$\ldots$ & $\ldots$ & $\ldots$ & $\ldots$ & $\ldots$ & $\ldots$ & $\ldots$ \\
\hline$S$. & $t_{i s}$ & $P_{1 S}$ & $P_{2 S}$ & $P_{3 S}$ & $\ldots$ & $P_{k S}$ \\
\hline
\end{tabular}

Fuzzification is performed during the next step i.e. the transformation of even values of input variables Pi into fuzzy $M_{i}$ using the linguistic variable. Such a transformation is in fact a kind of standardization necessary for the transfer of data into linguistic tags. Linguistic tags for the transfer of a clear meaning into fuzzy ones are stored in the knowledge base ASID. The result of the work at this stage is the generated parameter table, where fuzzy marks are placed instead of clear values. In general, the result of this stage is presented in Table 2 .

Table 2. Fuzzy Input Parameters

\begin{tabular}{|c|c|c|c|c|c|c|}
\hline № & Running time & Fuzzy set 1 & Fuzzy set 2 & Fuzzy set 3 & $\ldots$ & Fuzzy set $k$ \\
\hline 1. & $t=0$ & $M_{11}$ & $M_{21}$ & $M_{31}$ & $\ldots$ & $M_{k 1}$ \\
\hline 2. & $t=t_{\min }$ & $M_{12}$ & $M_{22}$ & $M_{32}$ & $\ldots$ & $M_{k 2}$ \\
\hline 3. & $t=t+t_{\min }$ & $M_{13}$ & $M_{23}$ & $M_{33}$ & $\ldots$ & $M_{k 3}$ \\
\hline & $\ldots$ & $\ldots$ & $\ldots$ & $\ldots$ & $\ldots$ & $\ldots$ \\
\hline$S$. & $t_{i S}$ & $M_{1 S}$ & $M_{2 S}$ & $M_{3 S}$ & $\ldots$ & $M_{k S}$ \\
\hline
\end{tabular}


The next step is the development of a fuzzy result in an output frame. To do this, fuzzy rules are applied from the "Knowledge Base" module. Then fuzzy input parameters of the engine are formed. With these rules, fuzzy output marks are generated (Table 3 ).

Table 3. Fuzzy Output Parameters

\begin{tabular}{|c|c|c|c|c|c|c|}
\hline$№$ & Running time & Fuzzy set 1 & Fuzzy set 2 & Fuzzy set 3 & $\ldots$ & Fuzzy set $k$ \\
\hline 1. & $t=0$ & $N_{11}$ & $N_{21}$ & $N_{31}$ & $\ldots$ & $N_{k 1}$ \\
\hline 2. & $t=t_{\min }$ & $N_{12}$ & $N_{22}$ & $N_{32}$ & $\ldots$ & $N_{k 2}$ \\
\hline 3. & $t=t+t_{\min }$ & $N_{13}$ & $N_{23}$ & $N_{33}$ & $\ldots$ & $N_{k 3}$ \\
\hline & $\ldots$ & $\ldots$ & $\ldots$ & $\ldots$ & $\ldots$ & $\ldots$ \\
\hline$S$. & $t_{i s}$ & $N_{1 S}$ & $N_{2 S}$ & $N_{3 S}$ & $\ldots$ & $N_{k S}$ \\
\hline
\end{tabular}

The next stage is dephasing. Dephasing means the procedure for fuzzy control action conversion into clear ones. Using them you can perform an engine

Table 4 - Control Actions

\begin{tabular}{|c|c|c|c|c|c|c|}
\hline Item № & Running time & Control Action 1 & Control Action 2 & Control Action 3 & $\ldots$ & Control Action $k$ \\
\hline 1. & $t=0$ & $y_{11}$ & $y_{21}$ & $y_{31}$ & $\ldots$ & $y_{k 1}$ \\
\hline 2. & $t=t_{\min }$ & $y_{12}$ & $y_{22}$ & $y_{32}$ & $\ldots$ & $y_{k 2}$ \\
\hline 3. & $t=t+t_{\min }$ & $y_{13}$ & $y_{23}$ & $y_{33}$ & $\ldots$ & $y_{k 3}$ \\
\hline & $\ldots$ & $\ldots$ & $\ldots$ & $\ldots$ & $\ldots$ & $\ldots$ \\
\hline$S$. & $t_{i s}$ & $y_{1 s}$ & $y_{2 s}$ & $y_{3 s}$ & $\ldots$ & $y_{k s}$ \\
\hline
\end{tabular}

The stages of fuzzification, derivation and defuzzification are carried out using the standard mathematical apparatus of fuzzy set theory.

The final stage is the testing of the engine based on the received clear control actions.

\section{Summary}

Thus, the application of a neuro-fuzzy system will shorten the time of the knowledge base development and will increase the adequacy of an engine control at different modes with the increase in the number of carried out engine tests. The use of the direct measurement method will also make it possible to apply the already available knowledge base of the tested engine to another engine of the same series.

The result of the work carried out is the software development for ASID engines.

\section{Acknowledgements}

The work is performed according to the Russian Government Program of Competitive Growth of Kazan Federal University.

\section{References}

1. M. Tian. Fuzzy neural network diagnose expert system of engine // ICICIP 2012 - 2012 3rd International Conference on Intelligent Control and Information Processing, 2012. - pp. 154-156.

2. Z.T. Yao, H.X. Pan. Engine fault diagnosis based on improved BP neural network with conjugate gradient // Applied Mechanics and Materials, 2014. - №536, pp. 296-299.

3. Valiev R.A., Galiullin L.A. Automated system of engine tests on the basis of Bosch controllers // International Journal of Applied Engineering Research, diagnostics and testing. The result of this step is shown in Table 4.
2015. - 10 (24). - pp. 44737-44742.

4. Galiullin L.A. Automated test system of internal combustion engines // (2015) IOP Conference Series: Materials Science and Engineering, 86 (1), art. no. 012018.

5. Valiev R.A., Galiullin L.A. Modeling of internal combustion engines test conditions based on neural network // International Journal of Pharmacy and Technology, 2016. - №8 (3). - pp. 14902-14910.

6. N. Deng, C.-S. Jiang. Fault diagnosis technology based on the fusion of neural network and fuzzy Logic // 2012 International Conference on Systems and Informatics, 2012. - pp. 419-422.

7. Z.T. Yao, H.X. Pan. Engine fault diagnosis based on improved BP neural network with conjugate gradient // Applied Mechanics and Materials, 2014. - №536-537. pp. 296-299.

8. Zubkov E.V., Galiullin L.A. Hybrid neural network for the adjustment of fuzzy systems when simulating tests of internal combustion engines // Russian Engineering Research, 2011. - №31 (5). - pp. 439-443. 9. R.A. Valiyev, L.A. Galiullin, A.N. Iliukhin. Design of the modern domain specific programming languages // International Journal of Soft Computing, 2015. №10 (5). - pp. 340-343.

10. R.A. Valiev, A.Kh. Khairullin, V.G. Shibakov. Automated Design Systems for Manufacturing Processes // Russian Engineering Research, 2015. №35 (9). - pp. 662-665. 